\title{
Early changes in ambulatory electrocardiography after transcatheter closure in patients with atrial septal defect and factors affecting heart rate variability
}

Zhenyang Su, Qing Cao, Hao Zhang* ${ }^{*}$, Wei Sun, Haifeng Zhang, Yanhui Sheng, Rong Yang and Xiangqing Kong

\begin{abstract}
Background: Factors affecting heart rate variability (HRV) in patients with atrial septal defect (ASD) have not been clarified. This study sought to identify those factors and establish a preliminary risk model.

Methods: A total of 154 patients with ASD who underwent transcatheter closure and met the study requirements were analyzed in this study. Moreover, 26 patients with patent foramen ovale (PFO) were enrolled in our study as a control group. All patients underwent echocardiography and ambulatory electrocardiography before and one day after the procedure.

Results: The standard deviation of all normal-to-normal (NN) intervals (SDNN) and the standard deviation of the averages of the NN intervals in all 5 min segments of the entire recording (SDANN) were significantly higher and the heart rate was lower after closure than before closure in patients with ASD (SDNN: 6.08, 95\% Cl 3.00 to 9.15, $p<0.001$; SDANN: $7.57,95 \% \mathrm{Cl} 4.50$ to $10.64, p<0.001$; heart rate: $-1.17,95 \% \mathrm{Cl}-2.86$ to $-0.48, p=0.006$ ). Multiple regression analyses indicated that age, sex, defect diameter, heart rate and diabetes were significantly associated with HRV indices (SDNN: $R^{2}=0.415 ; P<0.001$ ). SDNN and SDANN had obvious correlations with right ventricular systolic pressure (SDNN: $R=-0.370, p<0.001$; SDANN: $R=-0.360, p<0.001$ ).
\end{abstract}

Conclusions: Factors affecting HRV in patients with ASD include age, sex, heart rate, defect size and diabetes. Furthermore, right ventricular systolic pressure plays an important role in the change in HRV.

Keywords: Atrial septal defect, Ambulatory electrocardiography, Heart rate variability, Transcatheter closure

\section{Introduction}

Atrial septal defect (ASD) is one of the most common types of congenital heart disease. The interatrial defect results in a left-to-right shunt. It is generally accepted that defects smaller than $10 \mathrm{~mm}$ in diameter rarely result in changes in the heart structure [1]. When the defect diameter is large enough to cause significant hemodynamic changes, a longstanding shunt may result

\footnotetext{
* Correspondence: haozhanghh@163.com

Department of Cardiology, The First Affiliated Hospital of Nanjing Medical University, No. 300, Guangzhou Road, Nanjing 210029, China
}

in impaired heart structure and cardiac insufficiency. Atrial electrical remodeling resulting from chronic right atrial stretch in ASD patients frequently manifests as conduction delay at the crista terminalis and impaired sinus node function [2]. A recent study suggested that later arrhythmia can be predicted by measurement of left atrial volume [3].

Heart rate variability (HRV), which is known as a noninvasive, reliable indication of autonomic nervous system activity, has a unique manifestation in patients with ASD. It has been shown that HRV is reduced in children

C C The Author(s). 2020 Open Access This article is licensed under a Creative Commons Attribution 4.0 International License, which permits use, sharing, adaptation, distribution and reproduction in any medium or format, as long as you give appropriate credit to the original author(s) and the source, provide a link to the Creative Commons licence, and indicate if changes were made. The images or other third party material in this article are included in the article's Creative Commons licence, unless indicated otherwise in a credit line to the material. If material is not included in the article's Creative Commons licence and your intended use is not permitted by statutory regulation or exceeds the permitted use, you will need to obtain permission directly from the copyright holder. To view a copy of this licence, visit http://creativecommons.org/licenses/by/4.0/. The Creative Commons Public Domain Dedication waiver (http://creativecommons.org/publicdomain/zero/1.0/) applies to the data made available in this article, unless otherwise stated in a credit line to the data. 
with ASD [4, 5]. Surgery or transcatheter closure can improve HRV in pediatric patients [6-8]. However, there is not much research on HRV in adult patients with ASD. The factors that affect HRV in patients with ASD are not yet clear.

The aim of the present study was to evaluate the changes in HRV and heart rate in patients with ASD before and after transcatheter closure and explore factors affecting HRV indices in these patients.

\section{Methods}

\section{Study population and protocol}

The study population consisted of patients who underwent transcatheter closure of ASDs at the First Affiliated Hospital of Nanjing Medical University between October 2017 and December 2019. The exclusion criteria for this study were as follows: (1) concurrent presence of other congenital heart structural abnormalities, (2) diagnosis of serious atrial arrhythmias such as atrial fibrillation, atrial flutter and persistent atrial tachycardia before closure, (3) a history of acute myocardial infarction, or (4) receiving medications such as beta-blockers that could influence HRV measurements. During the same study period, patients with patent foramen ovale (PFO) device closure were enrolled in the study as the interventional control group. All patients underwent echocardiography and twenty-four-hour ambulatory electrocardiography before and 1 day after the procedure. All measurement data was recorded by two different blinded operators. The study protocol was approved by the Ethics Committee of the First Affiliated Hospital of Nanjing Medical University.

\section{Procedure of transcatheter closure}

The two groups of patients underwent an intervention with the same method: puncture the femoral vein, establishment of a delivery system and insertion of the device into the defect/oval foramen. Real-time two-dimensional transthoracic echocardiography was used for guidance during the process. Hemodynamic parameters were measured by a right heart catheter before closure in patients with ASD. Antiplatelet drugs were used in all patients after device implantation.

\section{Ambulatory electrocardiographic recordings and HRV indices}

Ambulatory electrocardiographic recordings were performed using an ambulatory 12-channel electrocardiogram (ECG) recorder. The recording time of all patients was not less than $23 \mathrm{~h}$. Mean heart rate and HRV indices were included in the study. The main indices of HRV included time-domain and frequency-domain indices. It has been confirmed that there is a strong correlation between time-domain and frequency-domain indices, and time-domain indices are more valuable and easy to analyze in the 24-h record [9-11]. Therefore, timedomain indices, which include the standard deviation of all normal-to-normal (NN) intervals (SDNN), the standard deviation of the averages of the $\mathrm{NN}$ intervals in all 5 min segments of the entire recording (SDANN), the square root of the mean of the sum of the squares of differences between adjacent NN intervals (RMSSD) and the percentage of adjacent NN intervals that differ from each other by more than $50 \mathrm{~ms}$ (pNN50), are the main research objects of HRV in our study. In particular, SDNN is taken as a dependable standard for medical stratification of cardiac risk when recorded over twentyfour hours $[10,12]$.

\section{Statistical analysis}

Continuous variables are expressed as the mean \pm standard deviation. Categorical variables are expressed as frequencies and percentages. All continuous variables obey normal distribution according to Kolmogorov-Smirnov test and Q-Q plot. Two-tailed two-sample t-test and paired t-test were used respectively to compare continuous variables between and within groups. Bonferroni correction was used to correct the significance level. The chisquare test was used to compare groups of categorical variables. Correlations were analyzed by using Pearson's correlation coefficient. Multiple linear regression analyses were performed to estimate factors influencing the HRV indices. Statistical significance was set at $p<0.05$. All statistical analyses were carried out using SPSS version 25.0 for Windows (SPSS Inc., Chicago, IL, USA). Statistical graphics were drawn with GraphPad Prism 7.0 for Windows (GraphPad Software Inc., San Diego, CA, USA).

\section{Results}

\section{Baseline characteristics}

The clinical characteristics of the enrolled ASD patients and PFO patients are presented in Table 1. No significant differences in age, sex ratio, body weight, blood pressure, hypertension or diabetes mellitus were found between the two groups. Defect diameters in patients

Table 1 Baseline Characteristics of the two groups of patients

\begin{tabular}{llll}
\hline Factor & $\begin{array}{l}\text { Group ASD } \\
(\boldsymbol{n}=\mathbf{1 5 4})\end{array}$ & $\begin{array}{l}\text { Group PFO } \\
(\boldsymbol{n}=\mathbf{2 6})\end{array}$ & $\boldsymbol{p}$ Value \\
\hline Age, years & $39.54 \pm 15.62$ & $40.58 \pm 14.22$ & 0.750 \\
Male sex, n (\%) & $43(27.9)$ & $5(19.2)$ & 0.357 \\
Weight, kg & $60.65 \pm 10.49$ & $60.59 \pm 11.09$ & 0.979 \\
Systolic BP, mmHg & $119.59 \pm 12.76$ & $120.23 \pm 13.71$ & 0.815 \\
Diastolic BP, mmHg & $75.74 \pm 9.23$ & $79.08 \pm 8.55$ & 0.087 \\
Hypertension, n (\%) & $34(22.1)$ & $3(11.5)$ & 0.297 \\
Diabetes, n (\%) & $6(3.9)$ & $1(3.8)$ & 1.000 \\
\hline
\end{tabular}


with ASD were measured through transesophageal echocardiogram before closure.

\section{Changes in ambulatory electrocardiography and transthoracic echocardiography parameters after transcatheter closure}

The data for ambulatory electrocardiography and transthoracic echocardiography before and after interventional therapy are summarized in Table 2. There were significant differences between the two groups in each HRV index before transcatheter closure. In the ASD group, the SDNN and SDANN were significantly higher and the mean heart rate was lower after closure than before closure (SDNN: 6.08, 95\% CI 3.00 to 9.15, $p<0.001$; SDANN: $7.57,95 \%$ CI 4.50 to $10.64, p<0.001$; heart rate: $-1.17,95 \% \mathrm{CI}-2.86$ to $-0.48, p=0.006)$. In the PFO group, all HRV indices, including SDNN, SDANN, RMSSD and PNN50, were significantly lower after closure than before closure, and the mean heart rate increased after closure (SDNN: $-29.62,95 \% \mathrm{CI}-42.05$ to $-17.18, p<0.001$; SDANN: $-22.89,95 \%$ CI -33.61 to 12.16, $p<0.001$; RMSSD: $-10.46,95 \%$ CI -33.61 to 12.16, $p<0.001$; PNN50: $-7.25,95 \%$ CI -10.24 to -4.26 , $p<0.001$; mean heart rate: $5.81,95 \%$ CI 2.70 to 8.92 , $p=0.001)$. In addition, there were immediate, significant changes in heart structure after transcatheter closure in patients with ASD. Right atrial diameter (RAD) and right ventricle diameter (RVD) were significantly lower than before (RAD: $-3.33,95 \% \mathrm{CI}-4.08$ to $-2.57, p<0.001$; RVD: $-3.20,95 \%$ CI -3.88 to $-2.53, p<0.001)$. Although the difference was small, left ventricular end diastolic diameter (LVEDD) increased significantly after transcatheter closure (LVEDD: 0.82, 95\% CI 0.32 to 1.31, $p=0.001)$. These changes were not significant in patients with PFO.

Patients with ASD were divided according to age stratification: $\leq 20$ years old, $21 \sim 40$ years old, $41 \sim 60$ years old, $\geq 60$ years old (Fig. 1). At the age of 20 years or younger, $21 \sim 40$ years and $41 \sim 60$ years, SDNN and SDANN increased significantly after closure. At the age of $41 \sim 60$ years, the mean heart rate reduced significantly after closure. Our data showed a trend of increased heart rate after closure at the age of 61 years or older, but the change did not reach statistical significance.

Factors affecting HRV in patients with ASD before closure To further understand factors affecting HRV in patients with ASD, linear regression and correlation analyses were performed (Fig. 2). SDNN and SDANN were the main research indicators of HRV. The impact of sex on HRV was compared. The values of SDNN and SDANN were significantly higher in male patients than in female patients. Linear regression and correlation analyses demonstrated that age, defect diameter and mean heart rate were significantly inversely correlated with SDNN and

Table 2 The ambulatory electrocardiography and transthoracic echocardiography data before and after closure

\begin{tabular}{|c|c|c|c|c|c|c|c|}
\hline & \multicolumn{3}{|c|}{ Group ASD $(n=154)$} & \multicolumn{3}{|c|}{ Group PFO $(n=26)$} & \multirow[t]{2}{*}{$\mathrm{PO}^{*}$} \\
\hline & Before & After & $p$ Value & Before & After & $p$ Value & \\
\hline \multicolumn{8}{|c|}{ HRV indices and HR } \\
\hline SDNN, ms & $98.70 \pm 24.08$ & $104.78 \pm 25.67$ & $<0.001$ & $134.08 \pm 33.80$ & $104.46 \pm 22.64$ & $<0.001$ & $<0.001$ \\
\hline SDANN, ms & $84.85 \pm 22.04$ & $92.42 \pm 25.11$ & $<0.001$ & $115.62 \pm 31.87$ & $92.73 \pm 22.57$ & $<0.001$ & $<0.001$ \\
\hline RMSSD, ms & $27.83 \pm 10.81$ & $26.21 \pm 10.73$ & 0.082 & $34.23 \pm 11.80$ & $23.77 \pm 10.97$ & $<0.001$ & 0.006 \\
\hline PNN50, \% & $8.08 \pm 7.74$ & $7.33 \pm 7.35$ & 0.262 & $13.10 \pm 9.89$ & $5.84 \pm 7.27$ & $<0.001$ & 0.004 \\
\hline $\mathrm{HR}$, bpm & $76.81 \pm 9.10$ & $75.14 \pm 9.06$ & 0.006 & $75.58 \pm 10.78$ & $81.38 \pm 8.17$ & 0.001 & 0.537 \\
\hline \multicolumn{8}{|c|}{ TTE parameters } \\
\hline$L A D, m m$ & $32.43 \pm 4.32$ & $31.92 \pm 4.54$ & 0.088 & $31.00 \pm 3.49$ & $29.58 \pm 4.49$ & 0.054 & 0.124 \\
\hline LVESD, mm & $27.95 \pm 2.98$ & $28.20 \pm 2.72$ & 0.199 & $29.54 \pm 2.21$ & $28.63 \pm 2.50$ & 0.055 & 0.013 \\
\hline LVEDD, mm & $42.81 \pm 4.03$ & $43.63 \pm 3.66$ & 0.001 & $45.38 \pm 3.70$ & $44.13 \pm 3.63$ & 0.042 & 0.004 \\
\hline $\mathrm{RAD}, \mathrm{mm}$ & $38.13 \pm 5.71$ & $34.80 \pm 4.58$ & $<0.001$ & $29.75 \pm 3.01$ & $28.88 \pm 3.22$ & 0.131 & $<0.001$ \\
\hline $\mathrm{RVD}, \mathrm{mm}$ & $38.92 \pm 5.01$ & $35.72 \pm 4.70$ & $<0.001$ & $29.17 \pm 3.32$ & $29.21 \pm 3.59$ & 0.957 & $<0.001$ \\
\hline LVEF, \% & $64.12 \pm 4.06$ & $65.00 \pm 2.92$ & 0.019 & $64.15 \pm 2.00$ & $64.53 \pm 4.04$ & 0.697 & 0.971 \\
\hline
\end{tabular}

"P0: $p$-values between the values prior to the procedure in the patient and control groups; $P$ value threshold of 0.017 was used after Bonferroni correction $\left(0.05^{*}(1 / 3)\right)$;

$P$ value threshold of 0.017 was used after Bonferroni correction $\left(0.05^{*}(1 / 3)\right)$; SDNN the standard deviation of all normal-to-normal (NN) intervals, SDANN the standard deviation of the averages of NN intervals in all 5 min segments of the entire recording, RMSSD the square root of the mean of the sum of the squares of differences between adjacent NN intervals, $p N N 50$ the percentage of adjacent NN intervals that differ from each other by more than 50 msm, $H R$ heart rate, $L A D$ left atrium diameter, $L V E S D$ left ventricular end systolic diameter, $L V E D D$ left ventricular end diastolic diameter, $R A D$ right atrium diameter, $R V D$ right ventricular diameter, LVEF left ventricular ejection fraction 


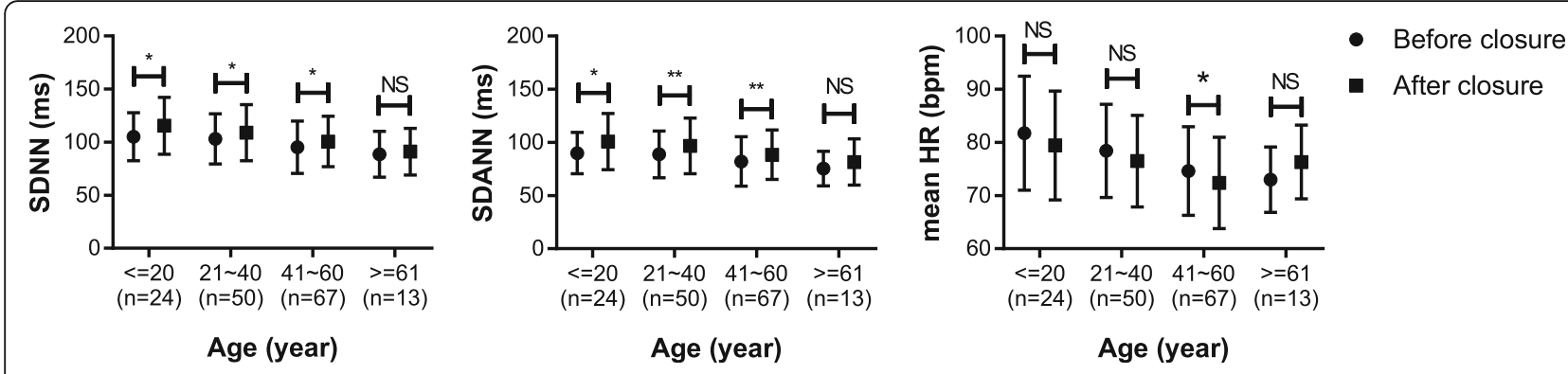

Fig. 1 Changes in SDNN, SDANN and mean heart rate before and after closure at different ages. Significance levels: NS, no significance; ${ }^{*} P<0.05$, ${ }^{*} P<0.01,{ }^{* * *} P<0.001$

SDANN. Additionally, diabetic patients had significantly lower SDNN values than nondiabetic patients. No significant differences in SDANN were found between diabetic and nondiabetic patients. Thus, multiple regression analyses indicated that female sex, old age, large defect diameter, fast heart rate and diabetes mellitus were predictors of HRV reduction. The regression equation was as follows (Table 3): SDNN (ms) $=258.94-11.42 \times[$ Sex $]-$ $0.49 \times$ [Age] $-1.09 \times$ [Defect diameter] $-1.32 \times$ [Mean heart rate] $-18.99 \times$ [Diabetes]. The $\mathrm{R}^{2}(0.415 ; P<0.001)$ value indicated that $41.5 \%$ of the variance in SDNN could be estimated using this model. In this equation, male sex is assigned a value of one, and female sex is assigned a value of two. The presence of diabetes is assigned one, and absence of diabetes is assigned zero.

Furthermore, after excluding patients younger than 20 years of age or with diabetes, correlations between HRV and pressure in the various chambers of the heart as measured by the right heart catheter were assessed (Fig. 3). SDNN and SDANN have obvious correlations with right ventricular systolic pressure (SDNN: $R=-0.370, p<$ 0.001; SDANN: $R=-0.360, p<0.001 ; n=124)$.
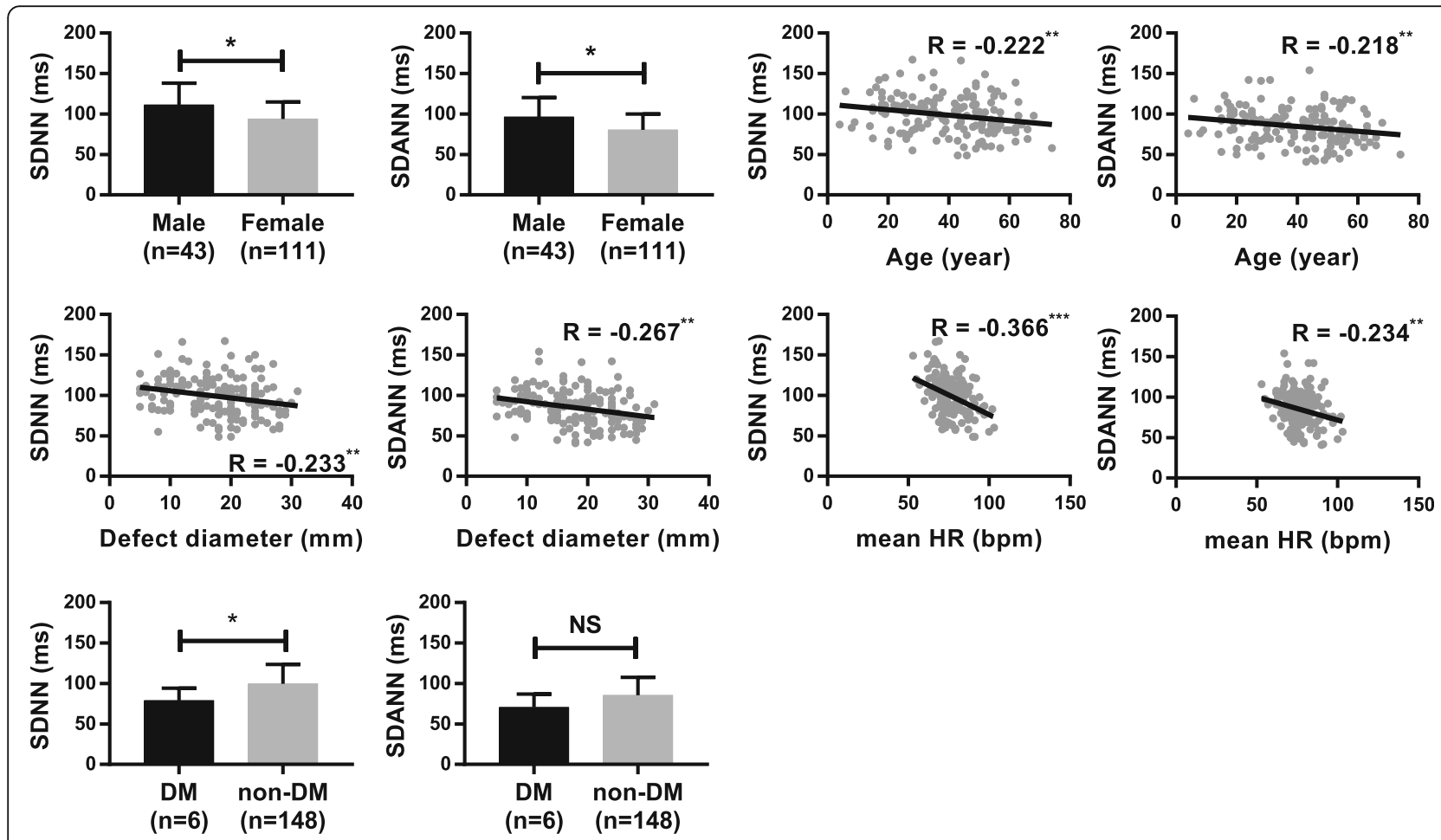

Fig. 2 Factors affecting HRV in patients with ASD. Comparison of SDNN and SDANN between different sexes. Linear regression and Pearson's correlation analyses showing the relationship of age, defect diameter and mean heart rate with SDNN and SDANN. Comparison of SDNN and SDANN between diabetic and nondiabetic patients. R, Pearson's correlation coefficient. Significance levels: NS, no significance; ${ }^{*} p<0.05$, ${ }^{* *} p<0.01,{ }^{* * *} p<0.001$ 
Table 3 Multiple linear regression analyses on SDNN and SDANN

\begin{tabular}{|c|c|c|c|c|c|c|}
\hline \multirow[t]{2}{*}{ Dependent variable } & \multirow[t]{2}{*}{ Variable } & \multicolumn{2}{|c|}{ Unstandardized coefficient } & \multirow[t]{2}{*}{ Standardized $\beta$} & \multirow[t]{2}{*}{$p$ Value } & \multirow[t]{2}{*}{$R^{2}$} \\
\hline & & $\bar{\beta}$ & Std. Error & & & \\
\hline \multirow[t]{5}{*}{ SDNN } & Age & -0.487 & 0.107 & -0.316 & $<0.001$ & 0.415 \\
\hline & Sex & -11.416 & 3.451 & -0.213 & 0.001 & \\
\hline & Defect diameter & -1.091 & 0.245 & -0.287 & $<0.001$ & \\
\hline & Heart rate & -1.316 & 0.181 & -0.498 & $<0.001$ & \\
\hline & Diabetes & -18.990 & 8.059 & -0.153 & 0.02 & \\
\hline \multirow[t]{4}{*}{ SDANN } & Age & -0.403 & 0.105 & -0.285 & $<0.001$ & 0.296 \\
\hline & Sex & -11.732 & 3.449 & -0.240 & 0.001 & \\
\hline & Defect diameter & -0.969 & 0.242 & -0.279 & $<0.001$ & \\
\hline & Heart rate & -0.840 & 0.181 & -0.347 & $<0.001$ & \\
\hline
\end{tabular}

SDNN the standard deviation of all normal-to-normal (NN) intervals, SDANN the standard deviation of the averages of NN intervals in all 5 min segments of the entire recording

\section{Discussion}

HRV, as a noninvasive examination to reflect the influence of sympathetic and vagal activity on the sinus node, is widely used in the diagnosis and prognostic assessment of clinical diseases. It has been confirmed that HRV is a strong and independent predictor of mortality in acute myocardial infarction [13]. Reduced HRV is also a marker of increased risk for mortality in healthy people without cardiac disease $[12,14]$. Changes in HRV in patients with ASD have also been reported.

Several studies have reported the effects of aging and sex on HRV [15-18]. While the linear relationship between HRV and age is controversial, there is no doubt that time-domain measurements of HRV decrease with age. Compared to men, women have lower HRV. In the present study, both SDNN and SDANN in males were significantly lower than those in females among patients with ASD. The values of SDNN and SDANN showed linear downward trends with age. This may be caused by a decrease in autonomic nerve activity with age.

The results of our study showed that SDNN and SDAN $\mathrm{N}$ decrease as the defect diameter increases. The size of the defect determines the scale of the left-to-right shunt. The overload of volume and pressure on the right heart caused by a longstanding shunt leads to chamber enlargement and elevated pulmonary artery pressure. Chronic right atrial stretch could cause sinus node dysfunction [2]. This may reduce the reactivity of the sinus node to autonomic nerve activity. Persistent pressure overload results in myocardial impairment and pulmonary hypertension [19-21]. It has been reported that patients with pulmonary hypertension have a marked alteration in cardiac autonomic activity [22]. Our study showed that SDNN and SDANN had negative linear correlations with right ventricular systolic pressure and pulmonary diastolic pressure. After transcatheter closure to block the shunt, the HRV increased, and the diameter of the right atrium and ventricle decreased. Changes in the right atrium and ventricular pressure and extension are intermediate factors in the process of HRV change. The control group comprised patients with PFO. Since there was no left-to-right shunt, the above situations were not observed in the control group.

The mean heart rate is a strong clinical determinant of HRV [12, 23]. Oliver et al. [23] noted that there is a universal exponential decay-like relationship between HRV and heart rate. Excessive resting heart rate could increase the risk of all-cause mortality [24]. The results of our study show that there was a negative linear
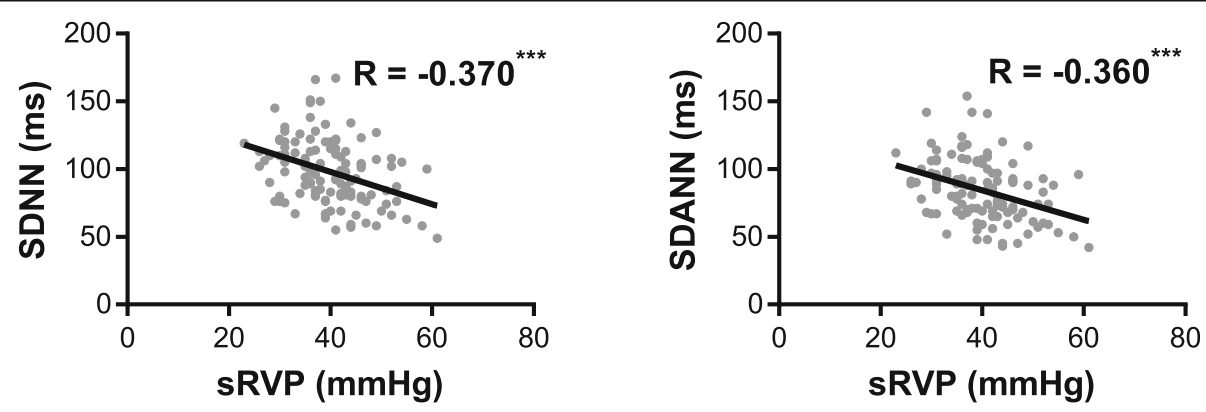

Fig. 3 Relationship between right ventricular systolic pressure and HRV. Linear regression and Pearson's correlation analyses showing the relationship of right ventricular systolic pressure (sRVP) with SDNN and SDANN. R, Pearson's correlation coefficient. Significance levels: NS, no significance; ${ }^{*} p<0.05,{ }^{* *} p<0.01,{ }^{* * *} p<0.001$ 
correlation between HRV and mean heart rate in patients with ASD, and improvement in HRV and reduction in heart rate appeared after closure. This may mean that transcatheter closure could reduce the risk of cardiac death in patients with ASD and improve prognosis.

Moreover, patients with diabetes or prediabetes have lower HRV [25, 26]. Diabetes-related cardiac autonomic dysfunction plays an important role in the decrease in HRV [27]. The results of our analyses show that having diabetes is associated with lower HRV, which also occurs in patients with ASD. In addition, our data did not show a linear relationship between blood pressure and HRV in patients with ASD, and there was no significant difference between HRV in hypertensive patients and HRV in non-hypertensive patients.

We included patients with PFO in the control group because both types of patients had congenital disorders of the atrial septum and both required the same procedure for interventional closure. The difference is that patients with ASD have obvious left-to-right shunts and significant changes in cardiac structure and function. There is almost no shunt in patients with PFO, so the postoperative changes are only related to the operation itself. Transcatheter closure could have a temporary negative effect on heart function. The specific manifestations of this effect were shown to be a decrease in HRV and an increase in heart rate after closure. This was a hypothesis and needs further research to confirm. Due to this effect, the immediate changes in HRV and HR of patients with ASD after transcatheter closure would be smaller than expected. This made the changes of HRV indices and HR after closure in patients with ASD statistically significant, but slight from a clinical perspective.

Therefore, we consider that the reduced HRV reflects the degree of heart damage caused by shunt. It is necessary to close the defects in patients with ASD in time, especially those with large defects. For patients who have not yet experienced a significant reduction in HRV, early closure is also beneficial, because age is also one of the factors affecting HRV. We believe that the above conclusions strengthen the indications for transcatheter closure. And postoperative changes in HRV can be used to evaluate the recovery of heart function, which shows the effectiveness and necessity of the percutaneous treatment.

\section{Study limitations}

Several limitations exist in this study. Because of the safety of the operation and the absence of short-term arrhythmias worthy of attention after transcatheter closure, most patients failed to complete follow-up ambulatory electrocardiograms. The follow-up time was not long enough in patients who underwent follow-up. Furthermore, HRV may also increase under pathological conditions, such as some special types of acute ischemic stroke [28]. Additionally, cerebral thromboembolism in patients with congenital heart disease is not uncommon, and these patients need more individualized attention.

Further research can be anticipated to expound the relationship between HRV and cardiac volume and intracavitary pressure. Therefore, HRV could be used to predict the long-term prognosis of patients after interventional closure and provide more sufficient evidence for the choice of closure timing. Whether the long-term changes in postoperative HRV in patients of different ages are significant is also a question worthy of further research.

\section{Conclusions}

Patients with ASD have significantly reduced HRV and increased heart rate, which can be improved by transcatheter closure. There is a negative correlation between HRV and heart rate. Other factors affecting HRV in patients with ASD include age, sex, diabetes, and defect size. Furthermore, right ventricular systolic pressure plays an important role in the change in HRV.

\section{Abbreviations}

ASD: Atrial septal defect; HRV: Heart rate variability; PFO: Patent foramen ovale; ECG: Electrocardiogram; SDNN: Standard deviation of all normal-tonormal (NN) intervals; SDANN: Standard deviation of the averages of NN intervals in all 5 min segments of the entire recording; RMSSD: Square root of the mean of the sum of the squares of differences between adjacent NN intervals; pNN50: Percentage of adjacent NN intervals that differ from each other by more than $50 \mathrm{~ms}$; HR: Heart rate; LAD: Left atrium diameter; LVES D: Left ventricular end systolic diameter; LVEDD: Left ventricular end diastolic diameter; RAD: Right atrium diameter; RVD: Right ventricular diameter; LVEF: Left ventricular ejection fraction

\section{Acknowledgements}

Not applicable.

\section{Authors' contributions}

$\mathrm{HZ1}$ and ZS conceived and designed the experiments. ZS collected the data, analyzed the data and drafted the manuscript. QC helped to review the data and the manuscript. $\mathrm{HZ1}$ helped to analyze the data and review the manuscript. WS, HZ2, YS, RY and XK helped to review the manuscript. All authors have read and approved the manuscript.

\section{Funding}

This work was supported by the Six Talent Peaks in Jiangsu Province (2014WSN-006).

Availability of data and materials

The datasets used and/or analyzed during the current study are available from the corresponding author on reasonable request.

Ethics approval and consent to participate

The study was approved by the Ethics Committee of the First Affiliated Hospital of Nanjing Medical University. Written informed consent was obtained from each patient.

Consent for publication

Not applicable.

Competing interests

The authors declare that they have no competing interests. 
Received: 12 July 2020 Accepted: 3 September 2020

Published online: 11 September 2020

\section{References}

1. Geva T, Martins JD, Wald RM. Atrial septal defects. Lancet. 2014;383:1921-32.

2. Morton JB, Sanders P, Vohra JK, Sparks PB, Morgan JG, Spence SJ, Grigg LE, Kalman JM. Effect of chronic right atrial stretch on atrial electrical remodeling in patients with an atrial septal defect. Circulation. 2003;107: 1775-82.

3. Duong P, Ferguson LP, Lord S, Murray S, Shepherd E, Bourke JP, Crossland D, O'Sullivan J. Atrial arrhythmia after transcatheter closure of secundum atrial septal defects in patients $\geq 40$ years of age. Europace. 2017;19:1322-6.

4. Massin MM, Derkenne B, von Bernuth $G$. Heart rate behavior in children with atrial septal defect. Cardiology. 1998;90:269-73.

5. Bakari S, Koca B, Oztunç F, Abuhandan M. Heart rate variability in patients with atrial septal defect and healthy children. J Cardiol. 2013;61:436-9.

6. Białkowski J, Karwot B, Szkutnik M, Sredniawa B, Chodor B, Zeifert B, Skiba A, Zyla-Frycz M, Kalarus Z. Comparison of heart rate variability between surgical and interventional closure of atrial septal defect in children. Am J Cardiol. 2003:92:356-8

7. Cansel M, Yagmur J, Ermis N, Acikgoz N, Taşolar H, Atas H, Muezzinoglu K, Pekdemir H, Ozdemir R. Effects of transcatheter closure of atrial septal defects on heart rate variability. J Int Med Res. 2011;39:654-61.

8. Özyılmaz İ, Ergül Y, Tola HT, Saygı M, Öztürk E, Tanıdır IC, Tosun Ö, Özyılmaz S, Gül M, Güzeltaş A, Ödemiş E, Bakır I. Heart rate variability improvement in children using transcatheter atrial septal defect closure. Anatol J Cardiol. 2016;16:290-5

9. Bigger JT, Fleiss JL, Steinman RC, Rolnitzky LM, Kleiger RE, Rottman JN. Correlations among time and frequency domain measures of heart period variability two weeks after acute myocardial infarction. Am J Cardiol. 1992; 69:891-8.

10. Malik M, Bigger J, Camm A, Kleiger RE, Malliani A, Moss AJ, Schwartz PJ. Heart rate variability: standards of measurement, physiological interpretation, and clinical use. Circulation. 1996;93:1043-65.

11. Koca B, Bakari S, Oztunç F. Correlation among the heart rate variability indices in healthy children and those with atrial septal defect. Turk Kardiyol Dern Ars. 2013:41:193-8.

12. Tsuji H, Venditti FJ, Manders ES, Evans JC, Larson MG, Feldman CL, Levy D. Reduced heart rate variability and mortality risk in an elderly cohort. The Framingham Heart Study. Circulation. 1994;90:878-83.

13. Kleiger RE, Miller JP, Bigger JT, Moss AJ. Decreased heart rate variability and its association with increased mortality after acute myocardial infarction. Am J Cardiol. 1987:59:256-62.

14. Dekker JM, Schouten EG, Klootwijk P, Pool J, Swenne CA, Kromhout D. Heart rate variability from short electrocardiographic recordings predicts mortality from all causes in middle-aged and elderly men. The Zutphen Study. Am J Epidemiol. 1997;145:899-908.

15. Tsuji H, Venditti FJ, Manders ES, Evans JC, Larson MG, Feldman CL, Levy D. Determinants of heart rate variability. J Am Coll Cardiol. 1996;28:1539-46.

16. Bonnemeier H, Richardt G, Potratz J, Wiegand UK, Brandes A, Kluge N, Katus HA. Circadian profile of cardiac autonomic nervous modulation in healthy subjects: differing effects of aging and gender on heart rate variability. J Cardiovasc Electrophysiol. 2003;14:791-9.

17. Almeida-Santos MA, Barreto-Filho JA, Oliveira JL, Reis FP, da Cunha Oliveira CC, Sousa AC. Aging, heart rate variability and patterns of autonomic regulation of the heart. Arch Gerontol Geriatr. 2016;63:1-8.

18. Koenig J, Thayer JF. Sex differences in healthy human heart rate variability: a meta-analysis. Neurosci Biobehav Rev. 2016;64:288-310.

19. Sugimoto M, Ota K, Kajihama A, Nakau K, Manabe H, Kajino H. Volume overload and pressure overload due to left-to-right shunt-induced myocardial injury. -evaluation using a highly sensitive cardiac troponin-I assay in children with congenital heart disease. Circ J. 2011;75:2213-9.

20. Steele PM, Fuster V, Cohen M, Ritter DG, McGoon DC. Isolated atrial septal defect with pulmonary vascular obstructive disease--long-term follow-up and prediction of outcome after surgical correction. Circulation. 1987;76: 1037-42.

21. Sachweh JS, Daebritz SH, Hermanns B, Fausten B, Jockenhoevel S, Handt S, Messmer BJ. Hypertensive pulmonary vascular disease in adults with secundum or sinus venosus atrial septal defect. Ann Thorac Surg. 2006:81: 207-13.
22. Wensel $R$, Jilek C, Dörr M, Francis DP, Stadler H, Lange T, Blumberg F, Opitz C, Pfeifer M, Ewert R. Impaired cardiac autonomic control relates to disease severity in pulmonary hypertension. Eur Respir J. 2009;34:895-901.

23. Monfredi $O$, Lyashkov $A E$, Johnsen $A B$, Inada $S$, Schneider $H$, Wang $R$, Nirmalan M, Wisloff U, Maltsev VA, Lakatta EG, Zhang H, Boyett MR. Biophysical characterization of the underappreciated and important relationship between heart rate variability and heart rate. Hypertension. 2014;64:1334-43.

24. Zhang D, Shen X, Qi X. Resting heart rate and all-cause and cardiovascular mortality in the general population: a meta-analysis. CMAJ. 2016;188:53-63.

25. Singh JP, Larson MG, O'Donnell CJ, Wilson PF, Tsuji H, Lloyd-Jones DM, Levy D. Association of hyperglycemia with reduced heart rate variability (the Framingham heart study). Am J Cardiol. 2000;86:309-12.

26. Coopmans C, Zhou TL, Henry RMA, Heijman J, Schaper NC, Koster A, Schram MT, van der Kallen CJH, Wesselius A, den Engelsman RJA, Crijns HJGM, Stehouwer CDA. Both prediabetes and type 2 diabetes are associated with lower heart rate variability: the Maastricht study. Diabetes Care. 2020;43:1126-33.

27. Vinik Al, Maser RE, Mitchell BD, Freeman R. Diabetic autonomic neuropathy. Diabetes Care. 2003;26:1553-79.

28. Shaffer F, Ginsberg JP. An overview of heart rate variability metrics and norms. Front Public Health. 2017:5:258.

\section{Publisher's Note}

Springer Nature remains neutral with regard to jurisdictional claims in published maps and institutional affiliations.
Ready to submit your research? Choose BMC and benefit from:

- fast, convenient online submission

- thorough peer review by experienced researchers in your field

- rapid publication on acceptance

- support for research data, including large and complex data types

- gold Open Access which fosters wider collaboration and increased citations

- maximum visibility for your research: over $100 \mathrm{M}$ website views per year

At BMC, research is always in progress.

Learn more biomedcentral.com/submissions 